\title{
Use of movement analysis in understanding abnormalities of gait in cerebral palsy
}

\author{
J H Patrick
}

Orthopaedic surgeons and paediatricians rarely use gait laboratory studies to assist decision making in the treatment of walking diplegic patients with cerebral palsy. This is now changing because of a realisation that operations for cerebral palsy can be vastly improved using such analysis.

An unlikely stimulus came in spring 1990 from the media. BBC television showed a film about the progress of a spastic boy from south London who visited a movement analysis laboratory at Newington Children's Hospital, Connecticut, USA. There he was assessed using VICON kinematics, electromyography, and forceplate measurements. He then underwent surgery and physiotherapy. The results of the operations were not spectacular, but the technology and the touching pictures of a courageous boy with cerebral palsy were more important than 'results'.

Doctors and therapists have been stimulated to think of improvements in diagnostic methods and ways of achieving a better understanding of the biomechanics of walking in individual patients. A case is made here for reconsidering the place of surgery for many patients with cerebral palsy by undertaking bony procedures and release or lengthening of tendons after gait analysis has been carried out, on more mature children aged from 7-8 years.

Although the television commentary did not mention the source of the equipment, that used in this patient's analysis was British; such systems are no longer just research tools, as is shown by the work of our laboratory. Accurate three dimensional data about limb movements in space can be obtained and viewed at leisure by rerunning video film after conventional examination of the patient has identified some of the problems in the lower limbs. Such equipment, together with synchronous electromyographic recordings of muscle activity and kinetic force estimations in the lower limb, can now be used to measure each ambulatory spastic child. Movement analysis is now more than an interesting research tool, because it permits accurate diagnosis of the causes and effects of deformity in cerebral palsy. Consequently, treatment possibilities suggest themselves: physiotherapy, orthoses, or operation.

Orthotic Research and Locomotor (ORLAU),

Robert Jones and

Agnes Hunt Orthopaedic Hospital,

Oswestry,

Shropshire SY10 7AG

J H Patrick

Correspondence to: Mr Patrick.
Traditional treatment regimen

For spastic children traditional methods of treatment have all included an assessment of walking potential. This is now a fine art, done best by skilled physiotherapists during a session of play, observation, and exercise usually undertaken during the first two years of life. In some areas of the country physiotherapists then remain at the forefront of physical management policy for that child. Orthopaedic surgeons and paediatricians usually accept this, as the attainment of walking is usually the province of physiotherapists and parents. Occasionally orthopaedic surgeons are asked to lengthen a tendon or muscle (usually at the groin or ankle), but the child grows and learns without much medical advice. Most children whose disability is confined to the legs, do walk; the more severely affected require wheelchairs, but there is a group for whom walking becomes increasingly difficult because of high energy costs of particular deformities which deleteriously affect walking.

Until recently we have been unable to analyse why some children apparently do well after operations, for example lengthening of the heel cord. Others, seemingly identical, adopt a

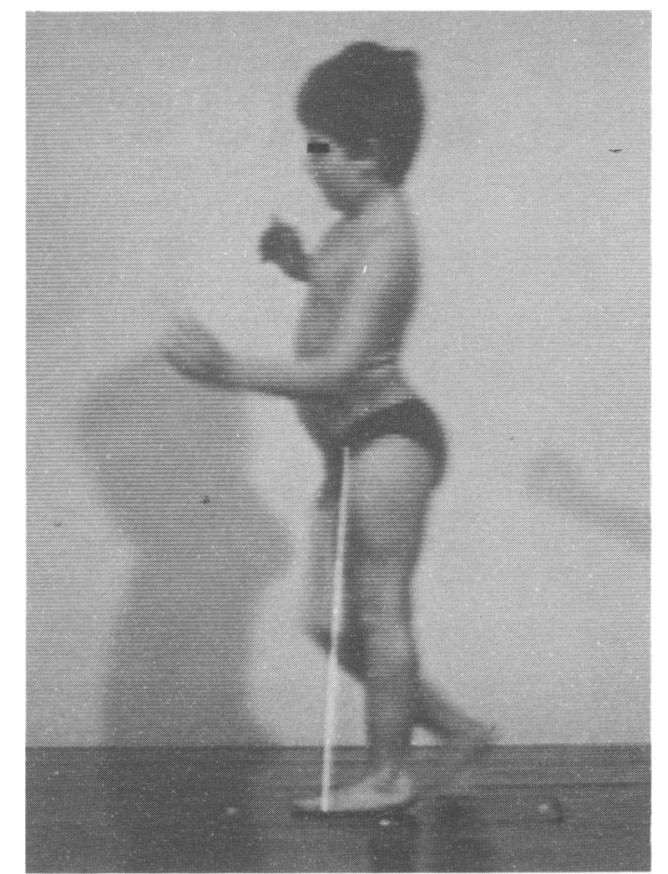

Figure 1 Left mid-stance phase in ORLAU movement analysis laboratory. Ground reaction vector line (representing body weight passing through force platform) shown as an extending moment to the left knee. Note slight equinus and lumbar lordosis caused by increased gastrocnemius and psoas lumbar lordosis caused by increased gastrocnemius and psoas
muscle tone. (Figure is a polaroid photograph taken from a moving video film.) 
crouch position after such operations. Why? Movement analysis now provides the answer (fig 1). A single frame photograph shows the body weight vector as a 'white line', accurately giving the position and direction of the body weight force as it is being applied to a Kistler force platform. Clearly, in this case, if the child has the heel cord lengthened then the ground reaction force vector may come to lie behind the knee (as in fig 2) unless the quadriceps can provide an opposing moment to straighten the knee. Often patients with cerebral palsy have quadriceps that are so weak that a 'crouch' is the only result of heel cord lengthening. This poor result (for some children) can now reliably be predicted, so the benefits of the analysis are explicit.

\section{Current problems}

Many children have heel cord operations that produce this 'crouch' position, because kinetic examinations such as ours are not generally available. The flexed knee and hip posture persists in spite of intense postoperative physiotherapy. Often surgical release of the flexion contracture of the knee is the next stage, and this has been called the 'birthday syndrome' because the child then undergoes still more physiotherapy, perhaps for another 6-12 months, until the surgeon releases the flexed hips of the 'crouch'. 'This last operation may destroy the flexor power of the limb (which is necessary to initiate the swing phase of gait) if the psoas tendon and muscle retract into the pelvis, their insertion into the femur having been lost. Surgical intervention is therefore not always a success and some patients are made cosmetically worse. Surgical treatment is

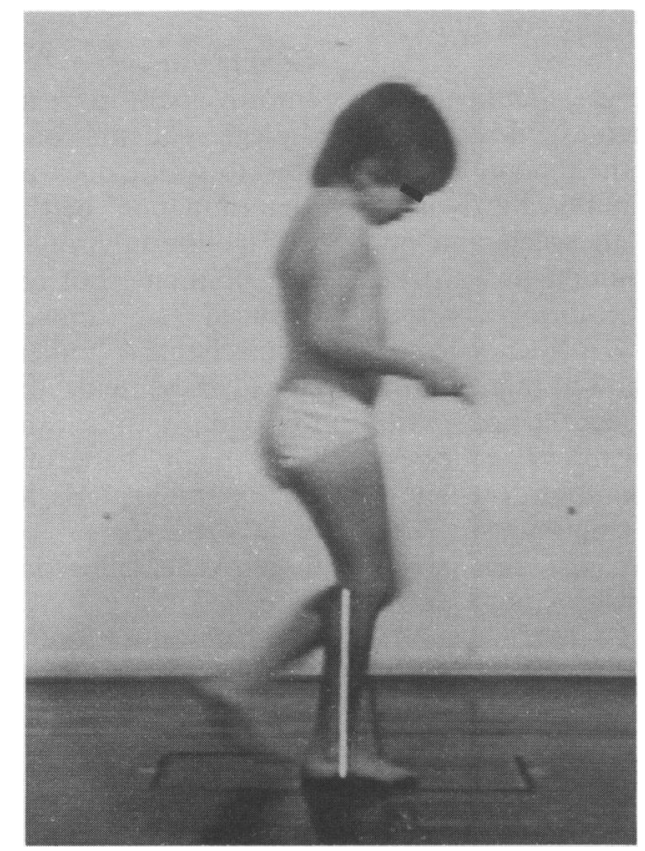

Figure 2 Right mid-stance on the force platform in ORLAU movement analysis laboratory. This girl has had her achilles tendon lengthened. The flexing moment to the knee is compensated for by quadriceps contraction-if this muscle weakens further she will 'crouch' by flexing hip and
mee is compensated for by quadriceps contraction-if this knee. (Figure is a polaroid photograph taken from a moving video film.) unfairly brought into disrepute if the biomechanics of that child are not known. Furthermore, neuromotor control is constantly maturing, and surgical intervention can be mistimed in growing children leading to bizarre gait problems in already handicapped spastic patients.

OTHER PROBLEMS IN MANAGEMENT OF DIPLEGIC CHILDREN

Sutherland and Hagys ${ }^{2}$ and Beals ${ }^{3}$ have pointed out that all diplegic children with cerebral palsy who can walk, mature at a slow but definite rate, following the developmental pattern of neuromuscular control in their normal peers, but less efficiently. By the age of 7 years both groups have matured maximally, so surgeons should if possible wait until the plateau of maximum control has been reached before operating. We propose that no operation should be undertaken until movement analysis has been made at the age of about 7-8 years. If a disabling dynamic deformity is present earlier than this, then it is probably the result of increased tone in the lower limbs. Oppenheim has shown that in some patients selective rhizotomy may be helpful, although experience of this is still limited. ${ }^{4}$

\section{Optimum management}

If clinical maturation levels off during the seventh year it should be easy to analyse which mechnical faults are present in an individual child. Sensible treatment is then possible. The gait analysis, preceded by traditional history taking and static examination, is followed by kinematic analysis to show how the child walks. We can note in slow motion the action and duration of the stance and swing phases, we can see objectively the limitations of movement in individual joints, and we can compare these with normal gait performance. Electromyographic examination may be helpful as cooperation is quite possible in this age group. Energy studies to assess the work being done have important prognostic value, as high values will suggest eventual limitation of activity and the necessity for confinement to a wheelchair.

\section{ANALYSIS OF LIMBS SEGMENTS}

In many diplegic children with cerebral palsy the hip flexors, hamstrings and rectus femoris muscles (at the front of the knee) are too tight; the Achilles tendon has increased tone, and the adductor musculature contributes to excessive internal rotation at the hip. The classic 'diplegic walk' is the result: the child moves on tiptoe with the knees permanently bent to about $40^{\circ}$, with relative fixity of knee and hip motion during the swing phase.

\section{Knees}

Gage et al have shown that stiff knees are often caused by inappropriate contraction of the rectus femoris muscle; dynamic electromyographs can be made in the gait laboratory to show this. If confirmed, release or transfer of the rectus 
muscle tendon just above the knee is suggested. Knee motion during the swing phase is restored and elaborate, energy wasting swinging of the limb from the hip (circumduction) is eliminated.

\section{Hips}

The internal rotation of the femur at the hips is conventionally said to be the result of adductor overactivity, but kinematic and electromyographic analysis has shown that often iliopsoas or gracilis tightness are responsible. It is salutary to speculate how many adductor releases and rotatory femoral osteotomies have been done without simultaneous muscle balancing procedures, or because of lack of understanding of the cause of the internal rotation. Kinematic study can show internal rotation occurring after initial foot contact under the influence of gracilis and medial hamstring overactivity. An electromyographic analysis will confirm the point and permit accurate diagnosis, with a distinction being made between this type of internal rotation and the adduction/internal rotation of true adductor muscle spasticity.

A component of the 'crouch gait' is a dynamic, end stance-phase hip flexion. This is usually caused by psoas muscle spasticity and is seldom noticed by the untrained eye. Static examination is often unhelpful, but kinematic and kinetic analysis can show simply the end of stance for, say, the left hip. If the iliopsoas muscle keeps the hip flexed at this moment, then left hip extension is prevented as the trunk moves forwards (with the swinging leg) over the stance hip. The result of this is a diminution of right step length, understandable by readers if they stand on the left leg keeping the left hip bent, and then reach forwards with the swinging right leg. The foot contact point ahead is shorter than if we stand normally and allow the left stance hip to extend.

The deforming psoas muscle should not be released from its lesser trochanteric insertion. If there is psoas over activity, the tendon can retract into the pelvis and the important dynamic function of the muscle (as an accelerator and initiator of hip and thigh motion forwards) is lost. This can be disabling for normal children; for those with cerebral palsy it greatly lessens their motor abilities. Gage showed that an intraabdominal, extraperitoneal lengthening of the psoas tendon at the pelvic brim overcomes this potential complication of operation, but allows hip extension so increasing step length. ${ }^{6}$ After the operation the physiotherapist and patient can develop this muscle action in a more normal fashion.

\section{Ankles}

Additional kinetic examinations can increase the understanding of mechanical problems in disabled walkers. A force plate examination carried out synchronously with the orthogonal walks can show the position of the ground reaction vector (fig 2 ). The length of the vector line represents the direction and quantity of the force. In the simplest example, altering the pitch of an ankle foot orthosis (below knee splint) can dramatically change the walk of a diplegic child. If the body weight vector is made to pass anterior to the knee, the weak quadriceps are strengthened. If the pitch is wrong, the ankle foot orthosis 'doesn't work', and the knee flexes more. This is confusing for child, parent, and orthotist. If the body weight forces are correctly distributed, walking is successful. These kinetic examinations can fine tune the ankle foot orthosis.

\section{Energy expenditure}

Surgery has been useful to many children with cerebral palsy, but measurement of the results has been inadequate. McGregor has shown that estimating changes in heart rate during walking and relating them to walking speed provides a simple, non-invasive and accurate method of evaluating treatment ${ }^{7} 8$ This has been confirmed by Rose et al. ${ }^{9}$ An index of the physiological cost (PCI) is derived in the laboratory during five timed walks on the gait track. The resting heart rate is subtracted from the exercising heart rate and divided by the speed. In our orginal group of nine patients with cerebral palsy who underwent movement analysis and then appropriate operation as indicated by the assessment (AV Nene, GA Evans. Spastic diplegia: a functional assessment of simultaneous multiple surgical procedures to assist walking. Paper presented to British Orthopaedic Association, September 1990.), we were pleased that eight had significant drops in their PCI.

This seems to confirm that if gait analysis is used preoperatively to indicate appropriate surgical management, then overall functional benefit occurs when these patients are reviewed two years or more after treatment is completed.

\section{Conclusion}

Improvement in treatment for quadriplegic and diplegic patients, of whatever ability, who are able to walk, does not depend solely on their 'determination' or their placement in different therapeutic environments, or even on therapists' opinions, but on certain mechanical facts deduced by movement analysis. If the biomechanical 'milieu' is abnormal then decisions can be made about how the mechanics of that child's walk can be improved. Much has been made here of surgical treatment, but clearly the analysis may suggest that splinting with orthoses or plaster, or physiotherapy targetted at one particular joint, is more appropriate.

Whatever the outcome of the assessment, a regimen of treatment can be suggested to the parents and therapists; it is important that the decision to proceed remains with them. We believe that this concept is new, is as yet unproved, but seems overwhelmingly sensible in theory. Longitudinal study of such patients is required, and represents a clear but identifiable opportunity to audit carefully the walking progress of a group of children in the expectation that progress is real, if our experience is to be believed. 
1 Rang M, Silver R, de la Garza J. Cerebral palsy. In: Lovell WW, Winter RB, eds. Pediatric orthopedics. Vol 1. 2nd Ed Philadephia: Lippincott, 1986:345-96.

2 Sutherland DH, Hagys JL. Measurement of gait movements from motion picture film. $\mathcal{J}$ Bone foint Surg 1990;54A 787-97.

3 Beals RK. Spastic paraplegia and diplegia: an evaluation of non-surgical and surgical factors influencing the prognosis for ambulation. F Bone foint Surg 1966;48A:827-46.

4 Oppenheim WL. Selective posterior rhizotomy for spastic cerebral palsy. Clin Orthop 1990;253:20-9.

5 Gage JR, Perry J, Hicks R, Koop S, Werntz JR. Rectus femoris transfer to improve knee function of children with femoris transfer to improve knee function of children with
cerebral palsy. Dev Med Child Neurol 1987;29:159-66.

6 Gage JR. Gait analysis for decision making in cerebral palsy. Gage JR. Gait analysis for decision making in cerebra
Bulletin Hosp ft Dis Orthop Inst 1983;43:147-63.

7 MacGregor J. The objective measurement of physical performance with long term ambulatory physiological surveillance equipment (LAPSE). In: Stott FD, Raftery EB, Goulding L, eds. Proceedings of 3rd International Symposium on Ambulatory Monitoring. London: Academic Press 1979:29-39.

8 MacGregor $\mathrm{J}$. The evaluation of patient performance using long-term ambulatory monitoring technique in the domiciliary environment. Physiotherapy 1981;67:30-3.

9 Rose J, Gamble JG, Burgos A, Medeiros J, Haskell WL. Energy expenditure index of walking for normal children and for children with cerebral palsy. Dev Med Child Neurol
1990;32:333-40.

\section{Commentary}

Gait laboratory analysis is a welcome attempt to introduce some science into a difficult and highly specialised area. Much orthopaedic energy has been directed to improve walking in children with spastic diplegia or hemiplegia. Even in the most experienced hands the results of surgery after careful clinical assessment are sometimes unpredictable and undesired.

Some reasons are not far to find. For example, intoeing is usually due to over activity of the medial hamstrings-and is corrected by lateral transfer of the semitendinosus. However, the psoas or other hip internal rotator may be responsible. The distinction can be made by analysis of the electromyogram during the walking cycle. Similarly analysis of the function of muscles which cross two joints such as the rectus femoris can assist operative decisions. ${ }^{1}$ Normally the rectus is active in early swing to help hip flexion as well as to prevent excessive knee flexion. If the rectus is active in stance also, and the problem is excessive hip flexion in standing, proximal release is logical. If, however, there is activity after early swing and the problem is excessive extension leading to a stiff gait distal release or transfer is a better option.

It is still an article of faith that better planned surgery will yield better results. This is entirely reasonable as, unlike dystonia, weak muscles in spastic disorders remain weak after surgery. Over active muscles remain strong.

Great caution must be exercised, therefore, in extending this analysis to children with extrapyramidal involvement. Comparison of results of procedures based on gait analysis with results of clinically based procedures will not be easy but will be necessary to convince sceptics, and this includes funding authorities.

As with all such surgery, the role of a physiotherapist throughout is crucial.

The picture of practice that has emerged in the United States where several gait laboratories have developed considerable experience is for recommendations to be made to the referring clinician. It is for those who know the child and family best to decide with them whether in this particular child in his particular social and educational setting, this particular procedure will be appropriate. Clinical judgments have not been replaced by gait analysis. Instead, I hope they will become directed to more logically relevant questions.

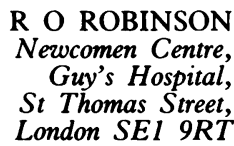

1 Perry J. Distal rectus femoris transfer. Dev Med Child Neurol 1987;27:153-8. 